\title{
Oral hygiene regimes for mechanically ventilated patients that use chlorhexidine reduce ventilator- associated pneumonia
}

\author{
Abstracted from \\ Shi Z, Xie H, Wang P, et al. \\ Oral hygiene care for critically ill patients to prevent ventilator-associated pneumonia. \\ Cochrane Database Syst Rev 2013; Issue 8. Art. No.: CD008367. DOI: 10.1002/14651858.CD008367.pub2. \\ Address for correspondence: Luisa Fernandez Mauleffinch, Review Group Co-ordinator, \\ Cochrane Oral Health Group, MANDEC, School of Dentistry, University of Manchester, \\ Higher Cambridge Street, Manchester, M15 6FH, UK. E-mail: luisa.fernandez@manchester.ac.uk
}

\section{Question: In patients receiving mechanical ventilation in intensive care units (ICUs) is oral health care $(\mathrm{OHC})$ effective in reducing ventilator-associated pneumonia (VAP)?}

Data sources The Cochrane Oral Health Group's Trials Register, the Cochrane Central Register of Controlled Trials (CENTRAL), Medline, Embase, CINAHL, LILACS, Chinese Biomedical Literature Database, China National Knowledge Infrastructure, Wan Fang Database, OpenGrey and ClinicalTrials.gov databases were searched. Reference lists of identified articles were also scanned for relevant papers. There were no language restrictions.

Study selection Randomised controlled trials (RCTs) evaluating OHC in the form of mouthwashes, swabs, toothbrushing or in combination in critically ill patients receiving mechanical ventilation were included. Data extraction and synthesis Data extraction was carried out independently by two reviewers. Study authors were contracted for additional information. Random-effects meta-analyses were performed where data could be pooled.

Results Thirty-five RCTs (5374 participants) were included. Five trials (14\%) were assessed at low risk of bias, 17 studies (49\%) were at high risk of bias and 13 studies (37\%) were assessed at unclear risk of bias in at least one domain. There were four main comparisons; chlorhexidine ( $\mathrm{CHX}$ mouthrinse or gel) versus placebo/usual care, toothbrushing versus no toothbrushing, powered versus manual toothbrushing and comparisons of oral care solutions.

Seventeen RCTs (2402 participants, two at high, 11 at unclear and four at low risk of bias) provide moderate quality evidence that $\mathrm{CHX}$ mouthrinse or gel, as part of $\mathrm{OHC}$, compared to placebo or usual care is associated with a reduction in VAP (OR $0.60,95 \%$ confidence intervals (Cl) 0.47 to $0.77, \mathrm{P}<0.001, \mathrm{I}^{2}=21 \%$ ) A number needed to treat (NNT) of 15 (95\% Cl 10 to 34). There is no evidence of a difference between $\mathrm{CHX}$ and placebo/usual care in the outcomes of mortality, duration of mechanical ventilation or duration of ICU stay. There was insufficient evidence to determine whether there is a difference between $\mathrm{CHX}$ and placebo/usual care in the outcomes of duration of use of systemic

This paper is based on a Cochrane Review published in the Cochrane Library 2013, issue 8 (see www.thecochranelibrary.com for information). Cochrane Reviews are regularly updated as new evidence emerges and in response to feedback, and the Cochrane Library should be consulted for the most recent version of the review. antibiotics, oral health indices, microbiological cultures, caregivers' preferences or cost. Only three studies reported any adverse effects, and these were mild with similar frequency in $\mathrm{CHX}$ and control groups.

Three trials in children found no evidence of a difference between $\mathrm{OHC}$ with $\mathrm{CHX}$ and placebo, and there was insufficient evidence to determine the effect of other outcomes. Four RCTs (828 participants, low quality evidence) compared to $\mathrm{OHC}$ without toothbrushing ( \pm $\mathrm{CHX}$ ), and for the outcome of VAP no evidence of a difference was found (OR $0.69,95 \% \mathrm{Cl} 0.36$ to $1.29, \mathrm{P}=0.24, \mathrm{I}^{2}=64 \%$ ). There was insufficient evidence to determine the effect of other outcomes.

Only one trial compared use of a powered toothbrush with a manual toothbrush, providing insufficient evidence to determine the effect on any of the outcomes of this review.

A range of other oral care solutions were compared. There is some weak evidence that povidone iodine mouthrinse is more effective than saline in reducing VAP $\left(\mathrm{OR} 0.35,95 \% \mathrm{Cl} 0.19\right.$ to $0.65, \mathrm{P}=0.0009, \mathrm{I}^{2}$ $=53 \%$ ) (two studies, 206 participants, high risk of bias). Due to the variation in comparisons and outcomes among the trials in this group there is insufficient evidence concerning the effects of other oral care solutions on the outcomes of this review.

Conclusions Effective $\mathrm{OHC}$ is important for ventilated patients in intensive care. $\mathrm{OHC}$ that includes either chlorhexidine mouthwash or gel is associated with a $40 \%$ reduction in the odds of developing ventilator-associated pneumonia in critically ill adults. However, there is no evidence of a difference in the outcomes of mortality, duration of mechanical ventilation or duration of ICU stay. There is no evidence that $\mathrm{OHC}$ including both $\mathrm{CHX}$ and toothbrushing is different from $\mathrm{OHC}$ with $\mathrm{CHX}$ alone, and some weak evidence to suggest that povidone iodine mouthrinse is more effective than saline in reducing VAP. There is insufficient evidence to determine whether powered toothbrushing or other oral care solutions are effective in reducing VAP.

\section{Commentary}

Ventilator associated pneumonia (VAP) can be a life-threatening infection for those on mechanical ventilation in intensive care units (ICUs). Oral hygiene is one of a number of prevention strategies and there have been a number of previous reviews of this topic. ${ }^{1}$ The Alhazzani review (see page 89$)^{1}$ was published just prior to the release of this Cochrane review. This review differs from the Alhazzani in that it is broader, including a

wider range of oral hygiene care $(\mathrm{OHC})$ interventions in addition to toothbrushing, with oral swabbing and mouthrinses also included. 


\section{Table 1. GRADE Working Group* grades of evidence}

\begin{tabular}{l|l}
\hline Grade & Definition \\
\hline High quality & $\begin{array}{l}\text { Further research is very unlikely to change our } \\
\text { confidence in the estimate of effect }\end{array}$ \\
\hline Moderate quality & $\begin{array}{l}\text { Further research is likely to have an important } \\
\text { impact on our confidence in the estimate of effect } \\
\text { and may change the estimate }\end{array}$ \\
\hline Low quality & $\begin{array}{l}\text { Further research is very likely to have an important } \\
\text { impact on our confidence in the estimate of effect } \\
\text { and is likely to change the estimate }\end{array}$ \\
\hline Very low quality & We are very uncertain about the estimate \\
\hline *http://www.gradeworkinggroup.org/
\end{tabular}

This review is typically well-conducted, following established Cochrane methodology. The key finding of this review was that inclusion of chlorhexidine mouthwash or gel as part of the oral health care regime showed a $40 \%$ reduction in the odds of developing VAP. This estimate remained very similar when a sensitivity analysis was conducted that excluded the high-risk studies, which found a very similar odds ratio (OR $0.61,95 \%$ CI 0.49 to $0.78, \mathrm{P}<$ $\left.0.001, \mathrm{I}^{2}=29 \%\right)$. The body of evidence supporting this was assessed as moderate using the GRADE methodology (Table 1)

Toothbrushing versus no toothbrushing did not show a difference in the incidence of VAP (OR 0.69, 95\% CI 0.36 to $1.29, \mathrm{P}=0.24$ ). This finding was very similar to Alhazzani who reported a trend towards lower VAP rates (risk ratio, 0.77; 95\% confidence interval, $0.50-1.21 ; \mathrm{P}=0.26$ ).

While the incidence of VAP was reduced, the available evidence did not reveal any positive effects on the other outcomes of mortality, duration of ventilation, length of stay in ICU, duration of antibiotic use. The authors discuss the mortality issue pointing out that a survival analysis by Bekaert et al. ${ }^{4}$ only found an attributable mortality of $1 \%$ at 30 days due to VAP, which is likely to explain the review's result.

In conclusion, effective oral care that includes the use of chlorhexidine should be a routine element of care for mechanically ventilated patents in ICU to reduce the incidence of VAP.

\section{Derek Richards} Centre for Evidence-based Dentistry, Oxford, UK

1. Alhazzani W, Smith O, Muscedere J, Medd J, Cook D. Toothbrushing for critically ill mechanically ventilated patients: a systematic review and meta-analysis of randomized trials evaluating ventilator-associated pneumonia. Crit Care Med 2013 41: 646-655. doi: 10.1097/CCM.0b013e3182742d45. Review. PubMed PMID: 23263588.

2. Pineda LA, Saliba RG, El Solh AA. Effect of oral decontamination with chlorhexidine on the incidence of nosocomial pneumonia: a meta-analysis. Crit Care 2006; 10: R35.

3. Labeau SO, Van de Vyver K, Brusselaers N, Vogelaers D, Blot SI. Prevention of ventilator-associated pneumonia with oral antiseptics: a systematic review and meta-analysis. Lancet Infect Dis 2011; 11: 845-854.

4. Bekaert M, Timsit JF, Vansteelandt S, et al. Attributable mortality of ventilatorassociated pneumonia: a reappraisal using causal analysis. Am J Respir Crit Care Med 2011; 184: 1133-1139.

Evidence-Based Dentistry (2013) 14, 91-92. doi:10.1038/sj.ebd.6400957 